\title{
Are behavioral interventions effective in increasing physical activity at 12 to 36 months in adults aged 55 to 70 years? A systematic review and meta-analysis
}

Nicola Hobbs ${ }^{1 *}$, Alan Godfrey², Jose Lara ${ }^{3}$, Linda Errington ${ }^{4}$, Thomas D Meyer ${ }^{5}$, Lynn Rochester ${ }^{2}$, Martin White ${ }^{1,6}$, John C Mathers ${ }^{3}$ and Falko F Sniehotta ${ }^{1,6}$

\begin{abstract}
Background: Retirement represents a major transitional life stage in middle to older age. Changes in physical activity typically accompany this transition, which has significant consequences for health and well-being. The aim of this systematic review was to evaluate the evidence for the effect of interventions to promote physical activity in adults aged 55 to 70 years, focusing on studies that reported long-term effectiveness. This systematic review adheres to a registered protocol (PROSPERO CRD42011001459).
\end{abstract}

Methods: Randomized controlled trials of interventions to promote physical activity behavior with a mean/median sample age of 55 to 70 years, published between 2000 and 2010, were identified. Only trials reporting the longterm effect ( $\geq 12$ months) on objective or self-reported physical activity behavior were included. Trials reporting physiological proxy measures of physical activity were excluded. Meta-analyses were conducted when trials provided sufficient data and sensitivity analyses were conducted to identify potential confounding effects of trials of poor methodological quality or with attrition rates $\geq 30 \%$.

Results: Of 17,859 publications identified, 32 were included which reported on 21 individual trials. The majority of interventions were multimodal and provided physical activity and lifestyle counselling. Interventions to promote physical activity were effective at 12 months (standardized mean difference $(S M D)=1.08$, 95\% confidence interval $(\mathrm{Cl})=0.16$ to 1.99 , pedometer step-count, approximating to an increase of 2,197 steps per day; $\mathrm{SMD}=0.19,95 \% \mathrm{Cl}$ $=0.10$ to 0.28 , self-reported physical activity duration outcome), but not at 24 months based on a small subset of trials. There was no evidence for a relationship between intervention effectiveness and mode of delivery or number of intervention contacts; however, interventions which involved individually tailoring with personalized activity goals or provision of information about local opportunities in the environment may be more effective.

Conclusions: Interventions in adults aged 55 to 70 years led to long term improvements in physical activity at 12 months; however, maintenance beyond this is unclear. Identified physical activity improvements are likely to have substantial health benefits in reducing the risk of age-related illnesses. These findings have important implications for community-based public health interventions in and around the retirement transition.

Keywords: Physical activity, intervention, aging, systematic review, meta-analysis

\footnotetext{
* Correspondence: nicki.hobbs@ncl.ac.uk

'Institute of Health \& Society, Newcastle University, Baddiley-Clark Building,

Richardson Road, Newcastle upon Tyne, NE2 4AX, UK

Full list of author information is available at the end of the article
} 


\section{Background}

Physical inactivity is a leading cause of death and disease. Epidemiological evidence shows a relationship between physical activity (PA) and reduced risks of coronary heart disease, Type II diabetes and some cancers, as well as increasing life expectancy [1,2]. The prevalence of disease and disability increases with age, making PA promotion an important public health objective to mitigate the burden of age-related illness [2,3]. However, over $50 \%$ of adults in Europe and the USA do not achieve public health recommendations for levels of PA [4-6].

PA is a modifiable behavior that varies in relation to major life events and transitions $[7,8]$. Retirement represents a key transition which impacts on physical and social activities [9]. Cross-sectional and longitudinal cohort studies show that PA levels change during retirement; however, the direction and magnitude of changes are inconsistent [10-13]. Previous occupation, socioeconomic and social factors may be important determinants of PA levels during retirement [14-18]. As PA levels are susceptible to change in retirement, then the retirement transition represents an ideal opportunity to intervene to increase and sustain PA behavior and, in turn, encourage healthy aging.

We are interested in promoting PA behaviors of people in and around the retirement transition. The average age of retirement varies between nations and from one year to the next. Since the early 2000s, retirement age is increasing in industrialized nations [19]. In the UK between 2004 and 2010, the average age of retirement rose from 64 to 65 and 61 to 62 for men and women, respectively [20]. Thus, in order to investigate the effect of interventions to increase PA in adults of a likely retirement age, we are focusing on adults between the ages of 55 and 70 years. Evidence from systematic reviews of PA interventions in middle-aged to older adults show moderate effects of interventions on PA behavior in the short- to mid-term (mean effect size of 0.28 [21] and 0.19 [22]). More effective interventions identified by these reviews were interventions which provided professional guidance and on-going support [21] and behavioral rather than cognitive interventions [22]. However, the evidence of PA interventions in the long-term effect is limited with only a few studies with follow-up assessments beyond 12 months [22]. From a public health perspective, it is critical to know whether PA behavior change can be sustained in the long-term. This systematic review and meta-analysis aims to synthesize the evidence from randomized controlled trials (RCT) on the effectiveness of interventions to promote long-term PA change ( $\geq 12$ months) in adults aged 55 to 70 years.

\section{Methods}

\section{Study selection criteria and search strategy}

This systematic review adheres to a registered protocol [23] (see Additional file 1). Only RCTs of interventions assessing and reporting PA behavior $\geq 12$ months after randomization were included. Included trials assessed PA behavior using objective or self-report measures. Interventions were compared to a no-intervention, minimal or usual care intervention; or a different type of intervention. Included trials studied healthy participants or those 'at risk' of chronic disease with a mean or median age of 55 to 70 years. 'At risk' participants were reported as having at least one of the following disease risk factors: hypertension, impaired glucose tolerance, overweight/obese, hyperlipidaemia, dyslipidaemia, family history, metabolic syndrome or osteopenia. Publications of any language with an English language abstract and with a country of origin of one of the 'most developed countries' within the United Nations index [24] were considered for inclusion.

Trials with inadequate randomization were excluded as were trials involving participants who were institutionalized or recruited on the basis of taking a particular medication or having a pre-existing chronic or acute medical condition. Trials that only reported PA behavior earlier than 12 months after randomization, that only reported physiological proxy measures of PA as distinct from PA behavior, were laboratory-based exercise studies, or promoted high or elite performance training were also excluded.

Twelve electronic databases were searched for articles published between January 2000 and November 2010 (Medline; Embase; PsycInfo; Scopus; Web of Science; CINAHL; ASSIA; Cochrane Database of Systematic Reviews, CAB Abstracts, Conference Papers Index, WorldCat Dissertations database and Index to Theses). Search terms relating to PA, middle- to older-aged people and RCT were translated into a Medline search strategy (see Additional file 2), which was adapted for other databases (available on request). Reference lists of reviews of PA interventions were hand-searched. After removing duplicate publications, the title and abstract of each publication was screened independently by two reviewers. When eligibility could not be ascertained or when reviewers disagreed, the full text was screened. Full text publications were also screened by two reviewers $($ Kappa $=0.91)$ and a third reviewer was consulted to resolve discrepancies. Reference lists of included publications were searched for additional publications.

\section{Data extraction}

Data from each included publication were extracted by one reviewer and independently checked by another (Kappa $=0.86)$; a third reviewer was consulted to resolve discrepancies. Authors were contacted for missing data and to provide additional intervention material. Intervention content was coded in line with intervention reporting guidance [25] to identify the modes of delivery 
and intervention intensity and trial quality was assessed using the Cochrane risk of bias tool [26].

\section{Data analysis}

The primary outcome was PA behaviors. Intervention effects were assessed by grouping trials for meta-analysis by the method of assessing PA and according to the duration of follow-up measurement after randomization. Random effects models (Review Manager (RevMan), version 5.1, Copenhagen, Denmark) were used and standardized mean differences (SMD) or odds ratios (OR) were calculated depending on whether the measurement scale was continuous or dichotomous. When trials included multiple intervention arms, which compared a PA intervention arm with a dietary intervention arm, the PA intervention arm was compared to the 'no intervention, control' arm. When trials included multiple intervention arms, which compared different types of PA intervention, the arm with the most intensive intervention content was compared with the 'no intervention, control' arm. The most intensive intervention arm was defined as the arm with the greater number of 'active' intervention contacts, that is., contacts when the intervention was delivered rather than contacts when only measurements were taken. When the number of contacts was equal in the intervention arms, the most intensive arm was defined as the arm delivered by a human rather than an automated system; the arm providing the most different types of information, using the most different modes of delivery or intervention aids; or the arm with the most intense exercise prescription. The content of all intervention arms reported in a trial is described in Additional file 3.

Data from intention-to-treat analysis were used when reported. If trials assessed PA using multiple methods, data were included in each corresponding meta-analysis. Estimations for total PA were selected for analysis over specific PA domains (for example, total PA rather than leisure time PA). Authors were contacted to provide mean and standard deviation when only the median and range were reported. When trials reported change scores from baseline, final values were computed where possible or requested from authors. For each main-effect analysis, sensitivity analyses were conducted by excluding trials of poor methodological quality or with attrition rates $\geq 30 \%$. Possible publication bias was assessed using funnel plots and Egger's tests [27]. Narrative analyses were applied when trials did not report sufficient data for meta-analyses or when the method of PA assessment was not equivalent to that used in any other trial.

\section{Results}

Thirty-two publications were included which reported on 21 distinct trials. Of these 21 trials, 15 were included in the meta-analyses [28-42] and six trials were analyzed narratively [43-48]. Nine trials were reported in multiple publications. The Green Prescription trial [29] was reported in two other publications [49,50]; the Community Health Advice by Telephone trial [30] in one other publication [51]; the Woman on the Move through Activity and Nutrition trial [31] in one other publication [52]; the Women's Lifestyle Study [32] in one other publication [53]; the Finnish Diabetes Prevention Study [33] in three other publications [54-56]; the Keep Active Minnesota trial [34] in one other publication [57]; the trial by Opdenacker and colleagues [36] in one other publication [58]; the Vitalum trial [39] in one other publication [59]; and the Pre-diabetes Risk Education and Physical Activity Recommendations and Encouragement trial [42] in one other publication [60]. Figure 1 displays the number of publications included and excluded at each stage, and reasons for exclusion (see Additional file 4 for PRISMA checklist). The characteristics of included trials including a description of the population, intervention and outcomes are tabulated (see Additional file 3).

Five authors provided additional unpublished data needed for meta-analyses, including mean and standard deviation when only the median and range were published and final values when only change scores from baseline were published $[29,31,32,41,42]$. Twelve authors responded to the request for additional intervention material $[28,29,32,34,37-41,43-45]$, seven of whom provided additional material $[28,29,34,39,40,44,45]$. Unpublished material provided in languages other than English was translated into English using online services and/or native speakers $[28,39,45]$. Additional English language publications describing intervention content were obtained for six trials [61-66].

Sixteen interventions were delivered by health professionals $[28-38,42,44-47]$, one intervention by the researcher [39], one by the participant under instruction (that is, self-help) [40], and the intervention provider was unclear in three trials $[41,43,48]$. The delivery format was multimodal for 14 trials (that is, face-to-face individual basis and via the telephone and/or printed material [29,30,32,43,44,47]; face-to-face group basis and via the telephone and/or printed material $[34,45,46]$; face-to-face individual and group basis plus via the telephone or printed material $[33,36,38]$; via the internet and printed materials [41]; or via the telephone and printed material [39]); unimodal for four trials (that is, face-to-face individual only [28]; face-to-face group only $[31,42]$; or printed material only [40]); and it was unclear whether the format was face-to-face individual or group for three trials $[35,37,48]$.

The intervention settings included healthcare premises $[29,32,33,38,42,43,45,47]$, the participant's home [30,34, $36,39-41]$, healthcare premises and at home $[28,35]$, in a 


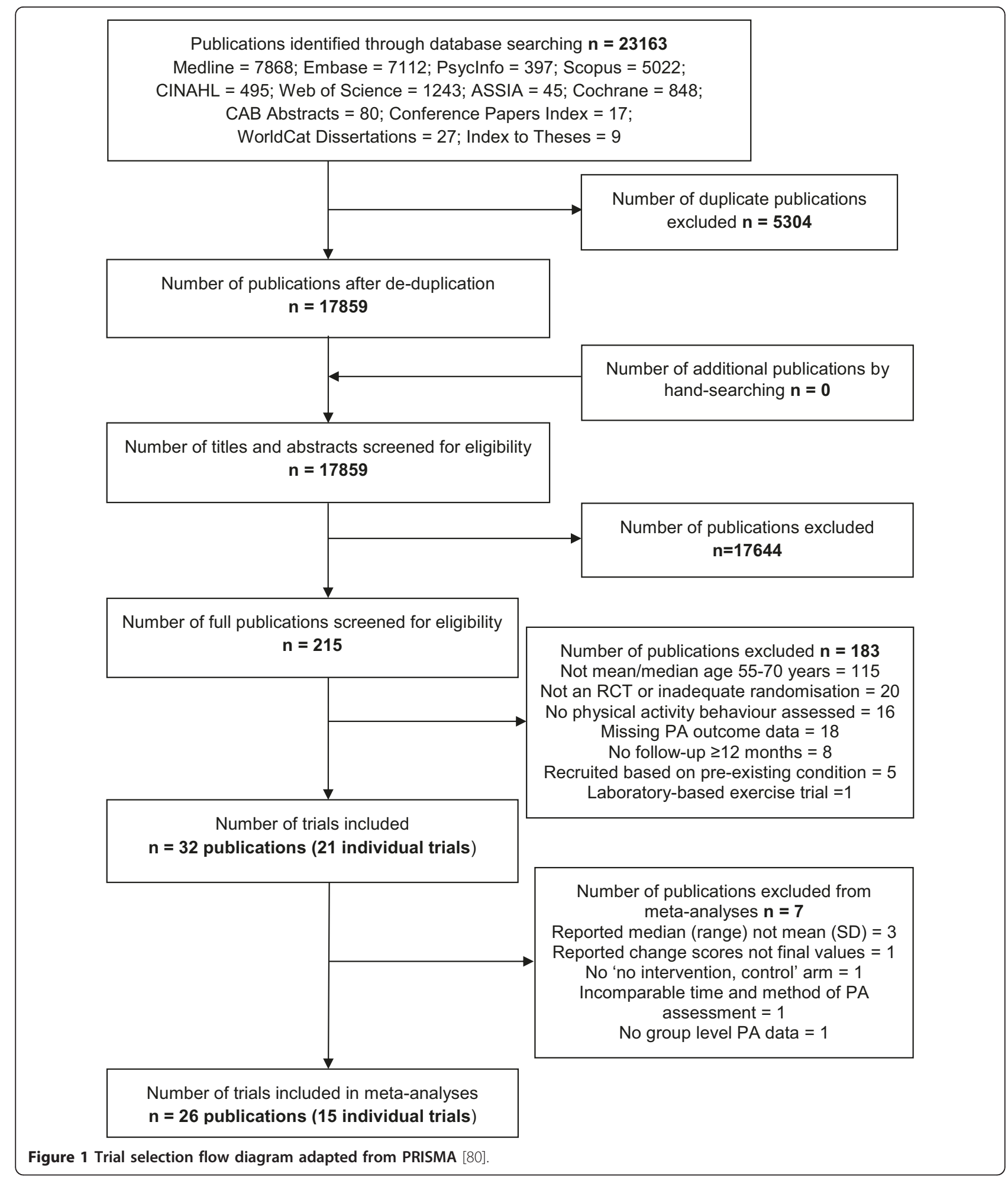

university facility [48], in a community setting [46] or this information was unspecified $[31,37,44]$. On average, trial length was 17 months from randomization $(\mathrm{SD}=$ 6.6), the 'active' intervention period was 8 months ( $\mathrm{SD}=$
4.6; range 1 to 11 ) with 37 contacts $(\mathrm{SD}=60$; range 1 to 228). The intervention period and the number of contacts were not specified in one trial [38]. Trials were conducted in the USA $[30,31,34,35,37,38,46,48]$, Belgium 
[36], The Netherlands [39-41,44,45], UK [42], Finland [33], New Zealand [29,32], Japan [28], Australia [43] and Canada [47]. Six trials used a clustered RCT design and accounted for clustering in analyses $[29,38,40,41,44,47]$.

All of the interventions, except one which involved group education and prescribed a standard PA goal of 150 minutes per week of moderately intense PA [31], were individually tailored to some degree. Five interventions provided participants with individually tailored exercise prescriptions, tailored on the basis of target heart rate $[35,45,48]$, submaximal $\mathrm{VO}_{2 \max }$ step test results [47] and baseline total energy expenditure [37]. The remaining interventions provided information specific to the individual to match their potential or actual health risk, their environment and local opportunities, and/or their individual PA goals. Thirteen of 21 interventions employed core self-regulation principles, such as goal setting, planning, self-monitoring and providing feedback [28-30,32-34,36,39-44].

In total, trials reported on 10,519 participants, $61 \%$ of whom were female. The mean age of participants was 60.7 years $(\mathrm{SD}=4.4$; range 55 to 67.6$)$. Two trials provided the intervention cost per participant $[28,29]$ but none reported a cost-effectiveness evaluation. Two trials specifically targeted participants in the retirement transition, namely retired employees [36] and recent retirees from a retirement workshop [41].

For many trials, there was insufficient information to permit conclusive judgements about methodological quality; the risk of bias was unclear in approximately $50 \%$ of possible judgements. Where judgements could be made, approximately $25 \%$ of trials were rated as poor quality attributable to a lack of blinding of participants or intervention personnel, missing outcome data or selective outcome reporting (see Additional file 5). Mild asymmetry was evident in the funnel plots; however, the results of Egger's test were not statistically significant (see Additional file 6).

\section{PA outcomes}

Six trials assessed PA objectively: five trials used pedometers deriving step-count $[28,31,35,36,42]$ and one trial used an accelerometer deriving vector magnitude [36]. Twenty trials estimated PA duration by self-report questionnaires reported as minutes of PA or energy expenditure (that is, kcal, kJ or metabolic equivalent (MET)) [29-48]. Four trials assessed PA using both objective and self-report methods $[31,35,36,42]$. Of the 15 trials included in the meta-analyses, four trials reported pedometer stepcount at 12 months $[28,35,36,42] ; 11$ trials reported PA duration at 12 months using a continuous measurement scale [29,30,32,34-37,39-42]; three trials reported PA duration at 12 months using a dichotomous measurement scale of the percentage of participants meeting a target PA duration [32,33,38]; two trials reported PA duration at 18 months using a continuous measurement scale [31,39]; and four trials reported PA duration at 24 months using a continuous measurement scale $[32,34,36,41]$. Seven trials were included in more than one meta-analysis [32,34-36,39,41,42].

\section{Pedometer step-count}

When compared with controls at 12 months, interventions had a significant positive effect on step-count (Figure 2: $\mathrm{SMD}=1.08,95 \% \mathrm{CI}=0.16$ to $1.99 ; I^{2}=95 \%$, $95 \%$ CI $=90$ to 97$)$. One additional trial [31] assessed step-count 18 months after randomization and similarly identified a significant intervention effect (SMD $=0.38$, $95 \% \mathrm{CI}=0.16$ to 0.60 ); however, this trial suffered from high attrition bias and did not report conducting intention-to-treat analysis. A single trial [36] with 24-month follow-up assessed step-count and found that the previously identified beneficial intervention effect on stepcount was not sustained $(\mathrm{SMD}=-0.01,95 \% \mathrm{CI}=-0.41$ to 0.40$)$.

\section{Accelerometer}

The single trial which reported PA using an accelerometer [36] did not identify a detectable effect of a lifestyle intervention on vector magnitude/week at 12 months (SMD = $0.18,95 \% \mathrm{CI}=-0.18$ to 0.55$)$ or at 24 months $(\mathrm{SMD}=$ $-0.01,95 \% \mathrm{CI}=-0.42$ to 0.40$)$.

\section{Self-reported PA duration}

Interventions had a significant small positive effect on PA duration at 12 months when measured on a continuous scale (Figure 3: SMD $=0.19,95 \% \mathrm{CI}=0.10$ to $0.28 ; I^{2}=41 \%, 95 \% \mathrm{CI}=0$ to 74$)$. Sensitivity analysis, removing trials with high attrition rates or low quality ratings $[37,40]$, did not alter the effect $(\mathrm{SMD}=0.18$, $95 \% \mathrm{CI}=0.07$ to $\left.0.29, I^{2}=52 \%\right)$. Interventions also had a positive effect on PA duration at 12 months when measured on a dichotomous scale (Figure 4: OR $=1.63$ $95 \% \mathrm{CI}=1.06$ to $2.49, I^{2}=84 \%$ ). The three studies meta-analyzed here used scales which quantified the percentage of participants meeting a target PA duration equating to meeting current PA recommendations.

Four additional trials that could not be meta-analyzed due to insufficient data, reported inconsistent intervention effects at 12 months. One trial [43] found that the intervention group reported more vigorous activity (minutes/ session) than the control group (intervention: median $=$ $20,25^{\text {th }}$ to $75^{\text {th }}$ percentile $=0$ to 35 ; control: median $=0$, $25^{\text {th }}$ to $75^{\text {th }}$ percentile $=0$ to $\left.15, P<0.05\right)$, but not more walking (intervention: median $=30,25^{\text {th }}$ to $75^{\text {th }}$ percentile $=10$ to 60 ; control: median $=30,25^{\text {th }}$ to $75^{\text {th }}$ percentile $=10$ to 60 ). Another trial [48] reported no change in self-reported PA from baseline to 12 months using the Physical Activity Scale for Elderly in response to either a diet and high exercise intervention, diet and low exercise intervention, or a diet-only intervention. The remaining 


\begin{tabular}{|c|c|c|c|c|c|c|c|c|c|c|}
\hline \multirow[b]{2}{*}{ Study or Subgroup } & \multicolumn{3}{|c|}{ Intervention } & \multicolumn{3}{|c|}{ Control } & \multicolumn{2}{|r|}{ Std. Mean Difference } & \multirow{2}{*}{\multicolumn{2}{|c|}{$\begin{array}{l}\text { Std. Mean Difference } \\
\text { IV, Random, } 95 \% \mathrm{Cl}\end{array}$}} \\
\hline & Mean & SD & Total & Mean & SD & Total & Weight & IV, Random, 95\% CI & & \\
\hline Babazono 2007 & 10373 & 4089 & 46 & 6815 & 3421 & 41 & $25.0 \%$ & $0.93(0.49,1.37)$ & & $\rightarrow-$ \\
\hline McTiernan 2007 & 9613 & 9626.2 & 100 & 6398 & 9722 & 102 & $25.9 \%$ & $0.33(0.05,0.61)$ & & $\square$ \\
\hline Opdenacker 2008 & 8689 & 493 & 55 & 7503 & 465 & 63 & $24.7 \%$ & $2.46(1.98,2.95)$ & & $\square$ \\
\hline Yates 2009 & 7640 & 2633.7 & 29 & 5824 & 3200.3 & 28 & $24.4 \%$ & $0.61(0.08,1.14)$ & & $\square-$ \\
\hline Total $(95 \% \mathrm{Cl})$ & & & 230 & & & 234 & $100.0 \%$ & $1.08(0.16,1.99)$ & & \\
\hline \multicolumn{9}{|c|}{ Heterogeneity: $\mathrm{Tau}^{2}=0.82 ; \mathrm{Chi}^{2}=57.23, \mathrm{df}=3(P<0.00001) ;\left.\right|^{2}=95 \%$} & $-2 \quad-1$ & $0 \quad 1$ \\
\hline \multicolumn{9}{|c|}{ Test for overall effect: $Z=2.30(P=0.02)$} & Favors control & Favors intervention \\
\hline
\end{tabular}

two trials reported increases in PA duration reported as MET hours/week (median difference $=13.9,95 \% \mathrm{CI}=$ 10.6 to 18.3 ) [45] and change in $\mathrm{kcal} / \mathrm{kg} /$ day (mean difference $=0.42,95 \% \mathrm{CI}=0.12$ to 0.72 ) [47] .

Beyond 12 months, there was little evidence for significant intervention effects. No intervention effect was identified on PA duration at 18 months when measured on a continuous scale $(\mathrm{SMD}=0.10,95 \% \mathrm{CI}=-0.08$ to 0.29 , $\left.I^{2}=55 \%\right)$ and similarly, the single trial which used a dichotomous scale [44] reported no effect of an individualized stage-matched intervention on PA duration (OR = $1.21,95 \% \mathrm{CI}=0.95$ to 1.54$)$; however, this trial was rated as poor quality. In addition, no positive intervention effect was identified by four trials assessing PA duration at 24 months using a continuous scale $(\mathrm{SMD}=0.07,95 \%$ $\mathrm{CI}=-0.06$ to $0.20, I^{2}=27 \%$ ) or by the single trial using a dichotomous scale $(\mathrm{OR}=1.33,95 \% \mathrm{CI}=1.03$ to 1.70$)$ [32]. In contrast to these null findings, the increase in MET hours/week identified at 12 months in response to a supervised group exercise intervention [45] was retained at 24 months in one trial (median difference $=4.1,95 \%$
$\mathrm{CI}=0.3$ to 8.3 ); however, this trial could not be meta-analyzed due to missing data. One trial [33] assessed PA at 36 months and reported no change in PA duration in the intervention group but detected an increase in moderateto-vigorous PA duration (minutes/week) (intervention: median change $=61$, IQR $=33$ to 168 ; control: median change $=6, \mathrm{IQR}=91$ to 104$)$.

\section{Trials comparing multiple interventions}

Seven trials compared multiple interventions at 12 months after randomization. The results of two high-quality trials suggest that mode of delivery is not necessarily important for intervention effectiveness. King and colleagues [30] compared telephone-assisted PA counseling by a trained health educator with telephone-assisted PA counseling by an automated telephone-linked computer system and concluded that both interventions were effective in increasing weekly PA when compared to a no intervention control $(\mathrm{SMD}=-0.07,95 \% \mathrm{CI}=-0.42$ to 0.28$)$. The large Vitalum trial of 1,600 participants [39] used a full factorial design comparing three different interventions and found that at 12 months, when compared to a no intervention control

\begin{tabular}{|c|c|c|c|c|c|c|c|c|c|c|}
\hline \multirow{3}{*}{$\begin{array}{l}\text { Study or Subgroup } \\
\text { Elley } 2003\end{array}$} & \multicolumn{2}{|c|}{ Intervention } & \multicolumn{4}{|c|}{ Control } & \multicolumn{2}{|r|}{ Std. Mean Difference } & \multirow{2}{*}{\multicolumn{2}{|c|}{$\begin{array}{r}\text { Std. Mean Difference } \\
\text { IV, Random, } 95 \% \mathrm{CI}\end{array}$}} \\
\hline & \multirow{2}{*}{$\begin{array}{r}\text { Mean } \\
2.24\end{array}$} & \multirow{2}{*}{$\begin{array}{r}\text { SD } \\
3.04\end{array}$} & \multirow{2}{*}{$\begin{array}{r}\text { Total } \\
451\end{array}$} & \multirow{2}{*}{$\begin{array}{r}\text { Mean } \\
1.67\end{array}$} & \multirow{2}{*}{$\begin{array}{r}\text { SD } \\
2.56\end{array}$} & \multirow{2}{*}{$\begin{array}{r}\text { Total } \\
427\end{array}$} & \multirow{2}{*}{$\begin{array}{r}\text { Weight } \\
16.2 \%\end{array}$} & \multirow{2}{*}{$\begin{array}{c}\text { IV, Random, } 95 \% \text { CI } \\
0.20(0.07,0.33)\end{array}$} & & \\
\hline & & & & & & & & & & $\multimap$ \\
\hline King 2007 & 165.8 & 136.8 & 61 & 112.3 & 136.8 & 62 & $4.8 \%$ & $0.39(0.03,0.75)$ & & \\
\hline Lawton 2008 & 144.05 & 170.04 & 120 & 113.06 & 151.37 & 75 & $6.7 \%$ & $0.19(-0.10,0.48)$ & & \\
\hline Martinson 2010 & 4163 & 2200.38 & 494 & 3941 & 2074.58 & 487 & $16.8 \%$ & $0.10(-0.02,0.23)$ & & - \\
\hline McTiernan 2007 & 298 & 489.4 & 100 & 68 & 489.4 & 102 & $7.0 \%$ & $0.47(0.19,0.75)$ & & \\
\hline Opdenacker 2008 & 4726 & 2410.3 & 55 & 4,751 & 2420.9 & 63 & $4.7 \%$ & $-0.01(-0.37,0.35)$ & & \\
\hline Racette 2008 & 14.3 & 4.2 & 18 & 10.7 & 4.1 & 10 & $1.1 \%$ & $0.84(0.03,1.65)$ & & $\longrightarrow$ \\
\hline van Keulen 2011 & 6.13 & 4.4 & 285 & 5.32 & 4.53 & 331 & $13.9 \%$ & $0.18(0.02,0.34)$ & & $\square$ \\
\hline van Stralen 2010 & 703.2 & 443.3 & 455 & 620 & 448 & 465 & $16.4 \%$ & $0.19(0.06,0.32)$ & & $\square$ \\
\hline Werkman 2010 & 135.6 & 53.5 & 164 & 137.5 & 52.8 & 164 & $9.9 \%$ & $-0.04(-0.25,0.18)$ & & \\
\hline Yates 2009 & 6387.9 & 6342.2 & 27 & 3059.4 & 3379.5 & 26 & $2.3 \%$ & $0.64(0.09,1.20)$ & & $\longrightarrow$ \\
\hline Total $(95 \% \mathrm{Cl})$ & & & 2230 & & & 2212 & $100.0 \%$ & $0.19(0.10,0.28)$ & & \\
\hline \multicolumn{9}{|c|}{ Heterogeneity: $\mathrm{Tau}^{2}=0.01 ; \mathrm{Chi}^{2}=17.02, \mathrm{df}=10(P=0.07) ; \mathrm{I}^{2}=41 \%$} & -0.5 & 0.5 \\
\hline \multicolumn{9}{|c|}{ Test for overall effect: $Z=4.28(P<0.0001)$} & Favors control & Favors intervention \\
\hline
\end{tabular}




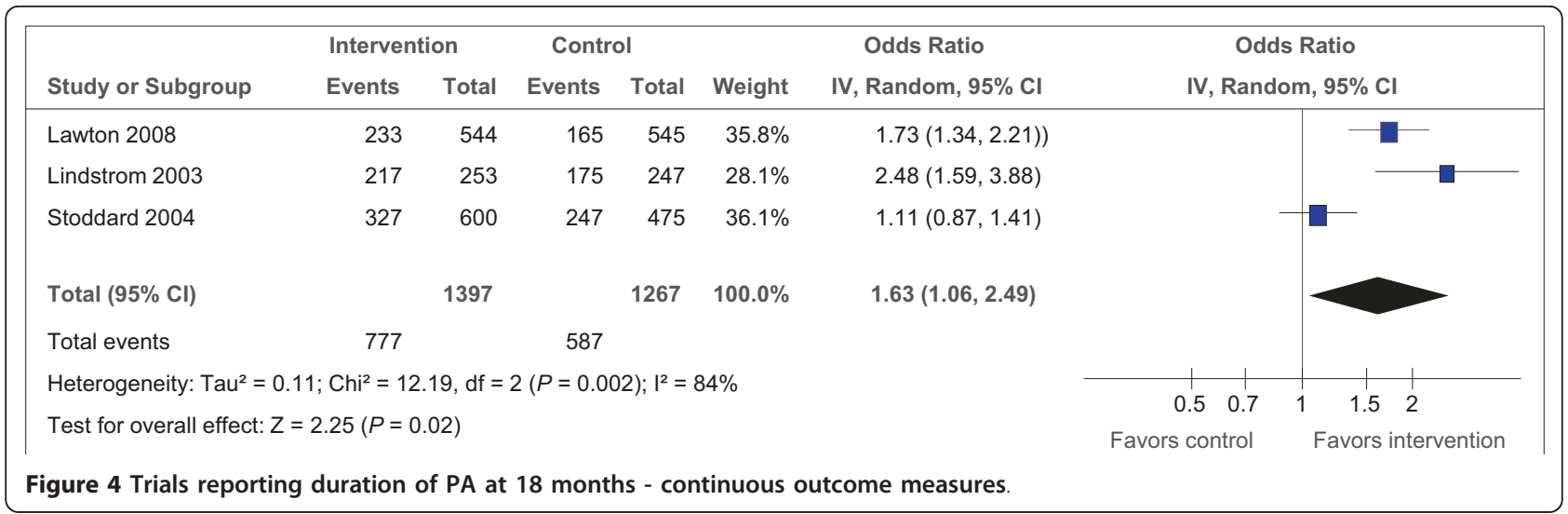

group, a tailored print intervention and a combined tailored print and motivational interviewing intervention produced improvements in self-reported PA (tailored print vs. control: $\mathrm{SMD}=0.32,95 \% \mathrm{CI}=0.15$ to 0.48 ; combined tailored print and motivational interviewing vs. control: $\mathrm{SMD}=0.18,95 \% \mathrm{CI}=0.02$ to 0.34 ), while a motivational interviewing-only intervention did not increase PA ( $\mathrm{SMD}=0.08,95 \% \mathrm{CI}=-0.08$ to 0.23 ). In addition, at 18 months, PA improvements were equally produced in response to all three interventions. These trials provide evidence to support the development of less resource intensive interventions that have greater potential to be cost-effective.

The type of PA that the intervention promotes may, however, be important. A trial of retired university employees [36] found that a home-based lifestyle intervention using a pedometer produced larger increases in active transport and daily steps at 12 months than a structured intervention involving supervised exercise sessions or a no intervention control (active transport, lifestyle vs. structured: SMD = $1.19,95 \% \mathrm{CI}=0.79$ to 1.59 ; lifestyle vs. control: $\mathrm{SMD}=$ $0.60,95 \% \mathrm{CI}=0.23$ to 0.97 ; daily steps, lifestyle vs. structured: $\mathrm{SMD}=2.49,95 \% \mathrm{CI}=2.00$ to 2.99 ; lifestyle vs. control: $\mathrm{SMD}=2.46,95 \% \mathrm{CI}=1.98$ to 2.95$)$. King and colleagues [46] found that an aerobic and strength training intervention resulted in greater self-reported daily energy expenditure (cal/kg/day) than a stretching and relaxation intervention; this trial only provided baseline-adjusted mean values, thus SMD cannot be calculated. A small trial of 26 participants [48] suggests that the intensity of the PA promoted is not an important factor for intervention effect; no difference in changes in PA was identified in response to a high intensity exercise prescription, a low intensity exercise prescription or a no intervention control.

Tailoring the intervention for the participant may be an important factor for producing positive intervention effects. A trial of almost 2,000 participants [40] found that when compared to a no intervention control group, an environmentally tailored intervention which provided personalized
PA advice and tailored information about opportunities in the environment resulted in an increase in total PA (SMD $=0.19,95 \% \mathrm{CI}=0.06$ to 0.32 ), while the 'basic' intervention which only provided personalized PA advice did not produce PA improvements $(\mathrm{SMD}=0.11,95 \% \mathrm{CI}=-0.02$ to 0.25). Similarly, in a trial of participants with impaired glucose tolerance, a tailored educational intervention using personalized step goals and a pedometer was more effective than an intervention using generic time-based goals and no pedometer, when compared to a no intervention control; the pedometer group produced larger increases in stepcount (tailored pedometer: $\mathrm{SMD}=0.61,95 \% \mathrm{CI}=0.08$ to 1.14; generic no pedometer: $\mathrm{SMD}=0.36,95 \% \mathrm{CI}=-0.18$ to 0.90 ) and self-reported walking (tailored pedometer: $\mathrm{SMD}=0.64,95 \% \mathrm{CI}=0.09$ to 1.20 ; generic no pedometer: $\mathrm{SMD}=0.29,95 \% \mathrm{CI}=-0.28$ to 0.85$)$ [42].

\section{Exploratory sub-group analysis}

The meta-analyses revealed heterogeneity [27]; therefore, potential reasons for this heterogeneity were probed based on intervention intensity. This secondary analysis only included trials of higher quality and was restricted to interventions on self-reported PA duration at 12 months measured on a continuous scale. Based on a median split, intervention effect was negatively associated with intervention intensity such that interventions that had more intervention contacts ( $\geq 11$ contacts) did not have a detectable intervention effect on PA duration $(\mathrm{SMD}=0.20$, 95\% CI $=$ -0.08 to $0.47, I^{2}=71 \%$ ) while interventions that had less intervention contacts ( $\ddot{\mathrm{E}}, 11$ contacts) had a positive intervention effect $\left(\mathrm{SMD}=0.16,95 \% \mathrm{CI}=0.06\right.$ to $0.27, I^{2}=$ $38 \%)$. However, there was no statistically significant difference between subgroups $\left(\mathrm{Chi}^{2}=0.05, P=0.82\right)$. The absence of a dose-response effect of interventions on PA duration at 12 months is displayed in a forest plot where trials are ordered by intervention intensity (Figure 5).

\section{Discussion}

This is the first systematic review to our knowledge to synthesize evidence from RCTs focusing on the long-term 


\begin{tabular}{|c|c|c|c|c|c|c|c|c|c|}
\hline \multirow[b]{2}{*}{ Study or Subgroup } & \multicolumn{3}{|c|}{ Intervention } & \multicolumn{3}{|c|}{ Control } & Std. Mean Difference & \multirow{2}{*}{\multicolumn{2}{|c|}{$\begin{array}{l}\text { Std. Mean Difference } \\
\text { IV, Random, } 95 \% \mathrm{CI}\end{array}$}} \\
\hline & Mean & SD & Total & Mean & SD & Total & IV, Random, $95 \% \mathrm{CI}$ & & \\
\hline Elley 2003 (1) & 2.24 & 3.04 & 451 & 1.67 & 2.56 & 427 & $0.20(0.07,0.33)$ & & 1 \\
\hline Yates 2009 (2) & 6387.9 & 6342.2 & 27 & 3059.4 & 3379.5 & 26 & $0.64(0.09,1.20)$ & & 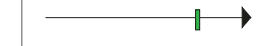 \\
\hline van Keulen 2011 (3) & 6.13 & 4.4 & 285 & 5.32 & 4.53 & 331 & $0.18(0.02,0.34)$ & & $\square$ \\
\hline Lawton 2008 (4) & 144.05 & 170.04 & 120 & 113.06 & 151.37 & 75 & $0.19(-0.10,0.48)$ & & 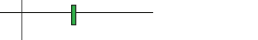 \\
\hline Martinson 2010 (5) & 4,163 & 2200.38 & 494 & 3941 & 2074.58 & 487 & $0.10(-0.02,0.23)$ & & $\square$ \\
\hline Werkman 2010 (6) & 135.6 & 53.5 & 164 & 137.5 & 52.8 & 164 & $-0.04(-0.25,0.18)$ & & $\mathbb{4}$ \\
\hline King 2007 (7) & 165.8 & 136.8 & 61 & 112.3 & 136.8 & 62 & $0.39(0.03,0.75)$ & & (2. \\
\hline Opdenacker 2008 (8) & 4726 & 2410.3 & 55 & 4751 & 2420.9 & 63 & $-0.01(-0.37,0.35)$ & & 4 \\
\hline \multirow[t]{2}{*}{ McTiernan 2007 (9) } & 298 & 489.4 & 100 & 68 & 489.4 & 102 & $0.47(0.19,0.75)$ & & $\square$ \\
\hline & & & & & & & & $\begin{array}{cc}-0.5 & -0.25 \\
\text { Favors control }\end{array}$ & $\begin{array}{lll}0 & 0.25 & 0.5 \\
\text { Favors intervention }\end{array}$ \\
\hline \multicolumn{10}{|l|}{ (1) 1 contact } \\
\hline \multicolumn{10}{|l|}{ (2) 1 contact } \\
\hline \multicolumn{10}{|l|}{ (3) 6 contacts } \\
\hline \multicolumn{10}{|l|}{ (4) 7 contacts } \\
\hline \multicolumn{10}{|l|}{ (5) 7 contacts } \\
\hline \multicolumn{10}{|l|}{ (6) 15 contacts } \\
\hline \multicolumn{10}{|l|}{ (7) 16 contacts } \\
\hline \multicolumn{10}{|l|}{ (8) 23 contacts } \\
\hline (9) 156 contacts & & & & & & & & & \\
\hline
\end{tabular}

effectiveness of interventions to promote PA in adults aged 55 to 70 years. We have provided evidence to show that PA interventions are effective at 12 months but this effect is not evident at 24 months. Based on a median split of the number of intervention contacts in high quality trials reporting continuous outcomes at 12 months, there is no evidence that more contacts lead to more favorable intervention effects. Evidence from trials comparing multiple interventions suggests that mode of delivery is not necessarily important for effectiveness but that tailoring the intervention to participants with personalized stepcount goals or information about local opportunities in the environment may be important.

\section{Findings in context and interpretation}

In a meta-analysis of four trials, we identified a large positive effect of 1.08 on step-count 12 months after randomization, approximating to an increase of 2,197 steps/day. The magnitude of this increase in step-count is similar to the 2,000 to 2,500 steps/day reported in previous metaanalyses of PA interventions lasting an average of 16 [67] and 18 [68] weeks. Our meta-analysis investigated the effect on step-count 12 months after randomization; therefore, this suggests that the improvements in stepcount acquired in the short to medium term can be sustained into the long-term.

In a meta-analysis of 11 trials, we identified an effect size of 0.19 on self-reported PA duration 12 months after randomization. This effect is smaller than previously reported in a meta-analysis of RCT with follow-up assessments at six months $(0.28)$ [21]. It is possible that intervention effects dissipate with time and perhaps the effects identified from trials with short or medium term followup assessments are not sustained in the longer-term. This hypothesis is supported by our finding that interventions were effective at 12 months but the benefits were not apparent at 24 months. Ten out of 11 trials assessing intervention effects on PA beyond 12 months consistently reported significant positive intervention effects. However, pooling trials for analysis by time and method of PA assessment did not identify positive intervention effects at 18 or 24 months. It is possible that the statistical power for each of these analyses was insufficient to detect an effect. Similarly, evidence from trials that could not be meta-analyzed did not provide clear support for positive intervention effects at 18,24 or 36 months with most trials reporting that intervention effects has dissipated beyond 12 months while others identified positive effects in some but not all methods of PA assessment.

Another recent meta-analysis [22] that aggregated interventions with objective and self-reported PA outcomes reported an effect size of 0.19 , which is the same size effect as we identified for self-reported outcomes. Our review adds to Conn and colleagues' review [22] by focusing on evidence of long-term effectiveness from RCT in adults aged 55 to 70 years. The average effect of 
PA interventions was higher on step-count than on selfreported PA duration. The accuracy of self-reported assessments of PA compared with objective assessments is uncertain; self-reported PA has been shown to overestimate and underestimate actual PA level [69]. Targeting specific activities, such as walking, may be more effective than targeting generic PA in people of retirement age. For example, one trial assessed PA using multiple methods and reported an increase in walking measured by a pedometer but no effect on PA measured by a selfreport questionnaire or accelerometer [36].

We found a lack of evidence for a relationship between intervention effectiveness and mode of delivery or intervention intensity in terms of the number of intervention contacts, which concurs with evidence from other reviews of physical activity interventions $[68,70]$. However, in line with previous literature $[68,71,72]$, there was evidence that using personalized step-count goals was better than using time-based goals, and that addressing physical environmental determinants of PA may be beneficial for PA promotion.

\section{Implications}

The substantial effect we identified on walking could produce important health benefits for older adults, such as improving weight-related outcomes, cardiorespiratory fitness, and cognitive and psychological well-being [73-77]. Furthermore, 100 steps per minute has been proposed to represent the floor value of moderate intensity walking [78]. The intensity of walking performed in the included trials is unclear; however, if the additional 2,197 steps per day were of moderate intensity, then this equates to approximately 22 minutes of moderate PA per day for an individual, which would contribute substantially towards meeting national PA recommendations of 150 minutes of moderate activity a week [6]. Moreover, a large recent prospective study of the health benefits of PA has shown that an additional 15 minutes of moderate PA per day may be sufficient to produce health benefits [79].

\section{Strengths and limitations}

The strengths of this review include that the search strategy was pre-specified and there was no statistical evidence that meta-analysis was affected by publication bias. Additional intervention material and data were requested from authors to improve the accuracy of the identification of intervention content and intervention intensity, and to limit missing data. We tested the sensitivity of our metaanalyses and found that the results were not influenced by trials with a high risk of bias. However, it is noted that the information provided in publications did not always allow conclusive judgements of methodological quality to be made, which resulted in many uncertain judgements. Thus, this observation limits the conclusiveness of these sensitivity analyses while highlighting the need for better reporting of trials.

This review only included trials which had been conducted with participants in countries categorized as being one of the 'most developed countries' within the United Nations index [24]. This inclusion criterion was chosen to ensure that the review focused on interventions that were applicable to populations experiencing broadly similar infrastructure, culture and standards of living. However, it is noted that, consequently, the findings of this review may not be generalizable to lower income countries. The included trials in this review had a sample mean or median age of 55 to 70 years, thus it is possible that the age of some individual participants included in this review may have been outside this bracket. However, 5 of 21 trials reported only recruiting participants within this age bracket and the average SD of the sample mean age in the remaining trials was seven years. Therefore, the relatively small variance in sample age suggests that in fact very few participants would not have been between 55 and 70 years old. In addition, the average age at retirement varies between individuals and nations, and varies over time [19]; therefore, adults aged 55 to 70 years are likely to reflect people in and around the retirement transition. Some people may have already retired, other people may be retiring soon, while other people may not have yet considered retiring.

\section{Conclusions}

The current evidence base is limited beyond 12 months and, therefore, RCT with longer follow-up are needed. Better reporting of trials is essential for complex interventions such as those included in this review. More factorial trials are needed to identify and isolate individual intervention components, such as tailoring and environmental factors, which may be associated with effectiveness.

\section{Additional material}

Additional file 1: Registered systematic review protocol. On inception of this systematic review, the protocol was registered with the National Institute of Health Research International Prospective Register of Systematic Reviews, PROSPERO: CRD42011001459.

Additional file 2: OVID Medline search strategy. Search terms and search strategy used to search for records in the OVID Medline electronic database.

Additional file 3: Table of characteristics of included trials in alphabetical order by author. Characteristics of the trials included in this review, including information on the study population, setting, physical activity outcome measures, assessment times, sample size in each intervention arm, content and delivery of intervention, and attrition rates.

Additional file 4: Preferred Reporting Items for Systematic Reviews and Meta-Analyses (PRISMA) 2009 checklist. Completed PRISMA 2009 
checklist detailing the page of the manuscript on which each checklist item is reported.

Additional file 5: Cochrane risk of bias figure. Risk of bias present in the trials included in this review based on the Cochrane risk of bias tool. Judgements about each risk of bias item are presented as percentages across the 21 included trials. The green bars represent the percentage of trials rated as high quality (low risk of bias) on each item, the yellow bars represent the percentage of trials where judgements could not be made (unclear risk of bias) on each item, and the red bars represent the percentage of trials rated as poor quality (high risk of bias) on each item.

Additional file 6: Funnel plots and Egger's test results assessing publication bias in the meta-analyses. Funnel plots and Egger's tests were used to identify the presence of publication bias in the metaanalyses. Publication bias could be tested for in the meta-analysis of trials reporting pedometer step-counts (steps/day) at 12 months; duration of physical activity at 12 months - continuous outcome measures; and duration of physical activity at 24 months - continuous outcome measures.

\section{Abbreviations}

Cl: confidence interval; PA: physical activity; RCT: randomized controlled trial; MET: metabolic equivalent; OR: odds ratio; SMD: standardized mean difference

\section{Authors' contributions}

$\mathrm{NH}, \mathrm{AG}, J \mathrm{~L}, \mathrm{TDM}, \mathrm{LR}, \mathrm{MW}, \mathrm{JCM}$ and FFS conceived and designed the study. LE performed the database searches. NH, AG, LR and FFS extracted the data. $\mathrm{NH}$ conducted data analysis and interpretation, and drafted the manuscript. $A G, J L, L R$, TDM, MW, JCM and FFS contributed to data interpretation. AG, JL, TDM, LE, LR, MW, JCM and FFS contributed to revising the manuscript. All authors approved the final manuscript. The corresponding author $(\mathrm{NH})$ had full access to all the data in the study and had final responsibility for the decision to submit the manuscript for publication.

\section{Competing interests}

The authors declare that they have no competing interests.

\section{Acknowledgements}

The authors thank Sue Lord for her help with data extraction, Suzanne McDonald for her help with coding intervention content, Satomi Miwa for translating Japanese intervention materials, and all authors who provided missing data and additional intervention materials.

This work is part of the LiveWell program. LiveWell is supported by the Lifelong Health and Wellbeing initiative (LLHW), which is a funding collaboration between the UK Research Councils and Health Departments. The LLHW funding partners are: Biotechnology and Biological Sciences Research Council, Engineering and Physical Sciences Research Council, Economic and Social Research Council, Medical Research Council, Chief Scientist Office of the Scottish Government Health Directorates, National Institute for Health Research/The Department of Health, The Health and Social Care Research and Development of the Public Health Agency (Northern Ireland), and Wales Office of Research and Development for Health and Social Care, Welsh Assembly Government. MW is partly and FFS fully funded by Fuse, the Centre for Translational Research in Public Health, a UKCRC Public Health Research Centre of Excellence. Funding for Fuse from the British Heart Foundation, Cancer Research UK, Economic and Social Research Council, Medical Research Council, and the National Institute for Health Research, under the auspices of the UK Clinical Research Collaboration, is gratefully acknowledged. Opinions expressed in this article do not necessarily represent those of the funders.

The funders had no role in study design, data collection and analysis, decision to publish, or preparation of the manuscript.

\section{Author details}

${ }^{1}$ Institute of Health \& Society, Newcastle University, Baddiley-Clark Building, Richardson Road, Newcastle upon Tyne, NE2 4AX, UK. ${ }^{2}$ Clinical Ageing Research Unit, Newcastle University, Campus for Ageing and Vitality, Newcastle upon Tyne, NE4 5PL, UK. ${ }^{3}$ Human Nutrition Research Centre,
Institute for Ageing and Health, Newcastle University, Biomedical Research Building, Campus for Ageing and Vitality, Newcastle upon Tyne, UK. ${ }^{4}$ Walton Library, Newcastle University, Newcastle upon Tyne, NE4 5PL, UK. ${ }^{5}$ Institute of Neuroscience, Newcastle University, Ridley Building, Newcastle upon Tyne, NE1 7RU, UK. ${ }^{6}$ Fuse, UKCRC Centre for Translational Research in Public Health, Institute of Health \& Society, Newcastle University, Baddiley-Clark Building, Richardson Road, Newcastle upon Tyne, NE2 4AX, UK.

Received: 19 December 2012 Accepted: 19 March 2013

Published: 19 March 2013

\section{References}

1. Kohl HW, Craig CL, Lambert EV, Inoue S, Alkandari JR, Leetongin G, Kahlmeier S: The pandemic of physical inactivity: global action for public health. Lancet 2012, 380:294-305.

2. Lee IM, Shiroma EJ, Lobelo F, Puska P, Blair SN, Katzmarzyk PT: Effect of physical inactivity on major non-communicable diseases worldwide: an analysis of burden of disease and life expectancy. Lancet 2012, 380:219-229.

3. World Health Organization: Global recommendations on physical activity for health. Geneva: World Health Organization; 2010.

4. Sjöström M, Oja P, Hagströmer M, Smith BJ, Bauman A: Health-enhancing physical activity across European Union countries: the Eurobarometer study. J Public Health 2006, 14:291-300.

5. Centers for Disease Control and Prevention: State Indicator Report on Physical Activity. Atlanta, GA: U.S. Department of Health and Human Services; 2010

6. Department of Health Physical Activity Health Improvement and Protection: Start active, stay active: a report on physical activity from the four home countries' Chief Medical Officers. London: Department of Health; 2011.

7. Brown WJ, Heesch KC, Miller YD: Life events and changing physical activity patterns in women at different life stages. Ann Behav Med 2009, 37:294-305.

8. Allender S, Hutchinson L, Foster C: Life-change events and participation in physical activity: a systematic review. Health Promot Int 2008, 23:160-172.

9. McMunn A, Nazroo J, Wahrendorf M, Breeze E, Zaninotto P: Participation in socially-productive activities, reciprocity and wellbeing in later life: baseline results in England. Age Soc 2009, 29:765-782.

10. Sjosten N, Kivimaki M, Singh-Manoux A, Ferrie JE, Goldberg M, Zins M, Pentti J, Westerlund $H$, Vahtera J: Change in physical activity and weight in relation to retirement: the French GAZEL Cohort Study. BMJ Open 2012, 2:e000522.

11. Evenson KR, Rosamond WD, Cai J, Diez-Roux AV, Brancati FL: Influence of retirement on leisure-time physical activity: the atherosclerosis risk in communities study. Am J Epidemiol 2002, 155:692-699.

12. Slingerland AS, van Lenthe FJ, Jukema JW, Kamphuis CB, Looman C Giskes K, Huisman M, Narayan KM, Mackenbach JP, Brug J: Aging, retirement, and changes in physical activity: prospective cohort findings from the GLOBE study. Am J Epidemiol 2007, 165:1356-1363.

13. Barnett I, van Sluijs EMF, Ogilvie D: Physical activity and transitioning to retirement: a systematic review. Am J Prev Med 2012, 43:329-336.

14. Chung S, Domino ME, Stearns SC, Popkin BM: Retirement and physical activity: analyses by occupation and wealth. Am J Prev Med 2009, 36:422-428.

15. Mein GK, Shipley MJ, Hillsdon M, Ellison GT, Marmot MG: Work, retirement and physical activity: cross-sectional analyses from the Whitehall II study. Eur J Public Health 2005, 15:317-322.

16. Shelton RC, McNeill LH, Puleo E, Wolin KY, Emmons KM, Bennett GG: The association between social factors and physical activity among lowincome adults living in public housing. Am J Public Health 2011, 101:2102-2110.

17. Carlson JA, Sallis JF, Conway TL, Saelens BE, Frank LD, Kerr J, Cain KL, King AC: Interactions between psychosocial and built environment factors in explaining older adults' physical activity. Prev Med 2012, 54:68-73.

18. Kouvonen A, De Vogli R, Stafford M, Shipley MJ, Marmot MG, Cox T, Vahtera J, Väänänen A, Heponiemi T, Singh-Manoux A, Kivimäki M: Social support and the likelihood of maintaining and improving levels of physical activity: the Whitehall II Study. Eur J Pub Health 2012, 22:514-518.

19. Banks J, Smith S: Retirement in the UK. Oxford Rev Econ Policy 2006, 22:40-56. 
20. Office for National Statistics: The labour market and retirement. Pension Trends Newport, UK: Office of National Statistics; 2012.

21. Foster C, Hillsdon M, Thorogood M: Interventions for promoting physical activity. Cochrane Database of Syst Rev 2005, CD003180.

22. Conn VS, Hafdahl AR, Mehr DR: Interventions to increase physical activity among healthy adults: meta-analysis of outcomes. Am J Public Health 2011, 101:751-758.

23. Hobbs N, Godfrey A, Sniehotta F, Rochester L, White M, Errington L, Mathers J: A systematic review of the maintenance effects of physical activity randomised controlled trials in healthy and 'at risk' older adults: LiveWell. PROSPERO: International Prospective Register of Systematic Reviews 2011, CRD42011001459.

24. United Nations Development Program: Human Development Report 2011. New York: United Nations Development Program; 2011.

25. Davidson KW, Goldstein M, Kaplan RM, Kaufmann PG, Knatterud GL, Orleans CT, Spring B, Trudeau KJ, Whitlock EP: Evidence-based behavioral medicine: what is it and how do we achieve it? Ann Behav Med 2003, 26:161-171.

26. In Cochrane handbook for systematic reviews of interventions. Edited by: Higgins JPT, Green S. The Cochrane Collaboration; 2009: Version 5.1.0 [Updated March 2011].

27. Higgins JP, Thompson SG, Deeks JJ, Altman DG: Measuring inconsistency in meta-analyses. BMJ 2003, 327:557-560.

28. Babazono A, Kame C, Ishihara R, Yamamoto E, Hillman AL: Patientmotivated prevention of lifestyle-related disease in Japan. A randomized controlled clinical trial. Dis Manage Health Outcomes 2007, 15:119-126.

29. Elley CR, Kerse N, Arroll B, Robinson E: Effectiveness of counselling patients on physical activity in general practice: cluster randomised controlled trial. BMJ 2003, 326:793.

30. King AC, Friedman R, Marcus B, Castro C, Napolitano M, Ahn D, Baker L: Ongoing physical activity advice by humans versus computers: the Community Health Advice by Telephone (CHAT) trial. Health Psychol 2007, 26:718-727.

31. Kuller LH, Kinzel LS, Pettee KK, Kriska AM, Simkin-Silverman LR, Conroy MB, Averbach F, Pappert WS, Johnson BD: Lifestyle intervention and coronary heart disease risk factor changes over 18 months in postmenopausal women: the Women On the Move through Activity and Nutrition (WOMAN study) clinical trial. J Womens Health (Larchmt) 2006, 15:962-974, Erratum: J Womens Health (Larchmt) 2006, 15:1101.

32. Lawton BA, Rose SB, Elley CR, Dowell AC, Fenton A, Moyes SA: Exercise on prescription for women aged 40-74 recruited through primary care: two year randomised controlled trial. BMJ 2008, 337:a2509.

33. Lindström J, Louheranta A, Mannelin M, Rastas M, Salminen V, Eriksson J, Uusitupa M, Tuomilehto J, Finnish Diabetes Prevention Study G: The Finnish Diabetes Prevention Study (DPS): lifestyle intervention and 3year results on diet and physical activity. Diabetes Care 2003, 26:3230-3236.

34. Martinson BC, Sherwood NE, Crain AL, Hayes MG, King AC, Pronk NP, $\mathrm{O}$ 'Connor PJ: Maintaining physical activity among older adults: 24-month outcomes of the Keep Active Minnesota randomized controlled trial. Prev Med 2010, 51:37-44.

35. McTiernan A, Sorensen B, Irwin ML, Morgan A, Yasui Y, Rudolph RE, Surawicz C, Lampe JW, Lampe PD, Ayub K, Potter JD: Exercise effect on weight and body fat in men and women. Obesity 2007, 15:1496-1512.

36. Opdenacker J, Boen F, Coorevits N, Delecluse C: Effectiveness of a lifestyle intervention and a structured exercise intervention in older adults. Prev Med 2008, 46:518-524.

37. Racette SB, Weiss EP, Schechtman KB, Steger-May K, Villareal DT, Obert KA, Holloszy JO: Influence of weekend lifestyle patterns on body weight. Obesity 2008, 16:1826-1830.

38. Stoddard AM, Palombo R, Troped PJ, Sorensen G, Will JC: Cardiovascular disease risk reduction: the Massachusetts WISEWOMAN project. $J$ Womens Health (Larchmt) 2004, 13:539-546.

39. van Keulen HM, Mesters I, Ausems M, van Breukelen G, Campbell M, Resnicow K, Brug J, de Vries H: Tailored print communication and telephone motivational interviewing are equally successful in improving multiple lifestyle behaviors in a randomized controlled trial. Ann Behav Med 2011, 41:104-118.

40. van Stralen MM, de Vries H, Bolman C, Mudde AN, Lechner L: Exploring the efficacy and moderators of two computer-tailored physical activity interventions for older adults: a randomized controlled trial. Ann Behav Med 2010, 39:139-150.

41. Werkman A, Hulshof PJ, Stafleu A, Kremers SP, Kok FJ, Schouten EG, Schuit AJ: Effect of an individually tailored one-year energy balance programme on body weight, body composition and lifestyle in recent retirees: a cluster randomised controlled trial. BMC Public Health 2010, 10:110.

42. Yates T, Davies M, Gorely T, Bull F, Khunti K: Effectiveness of a pragmatic education program designed to promote walking activity in individuals with impaired glucose tolerance: a randomized controlled trial. Diabetes Care 2009, 32:1404-1410.

43. Halbert JA, Silagy CA, Finucane PM, Withers RT, Hamdorf PA: Physical activity and cardiovascular risk factors: effect of advice from an exercise specialist in Australian general practice. Med J Aust 2000, 173:84-87.

44. Harting J, van Assema P, van Limpt P, Gorgels T, van Ree J, Ruland E, Vermeer $F$, de Vries NK: Cardiovascular prevention in the Hartslag Limburg project: effects of a high-risk approach on behavioral risk factors in a general practice population. Prev Med 2006, 43:372-378.

45. Hertogh EM, Vergouwe Y, Schuit AJ, Peeters PH, Monninkhof EM: Behavioral changes after a 1-yr exercise program and predictors of maintenance. Med Sci Sports Exerc 2010, 42:886-892.

46. King AC, Pruitt LA, Phillips W, Oka R, Rodenburg A, Haskell WL: Comparative effects of two physical activity programs on measured and perceived physical functioning and other health-related quality of life outcomes in older adults. J Gerontol (A Biol Sci Med Sci) 2000, 55:M74-83.

47. Petrella RJ, Lattanzio CN, Shapiro S, Overend T: Improving aerobic fitness in older adults: effects of a physician-based exercise counseling and prescription program. Can Fam Physician 2010, 56:e191-200.

48. Wang X, Lyles MF, You T, Berry MJ, Rejeski WJ, Nicklas BJ: Weight regain is related to decreases in physical activity during weight loss. Med Sci Sports Exerc 2008, 40:1781-1788.

49. Elley R, Kerse N, Arroll B, Swinburn B, Ashton T, Robinson E: Costeffectiveness of physical activity counselling in general practice. $N$ Z Med J 2004, 117:U1216.

50. Elley CR, Kerse N, Chondros P, Robinson E: Intraclass correlation coefficients from three cluster randomised controlled trials in primary and residential health care. Aust N Z J Public Health 2005, 29:461-467.

51. King AC, Friedman R, Marcus B, Castro C, Forsyth L, Napolitano M, Pinto B: Harnessing motivational forces in the promotion of physical activity: The Community Health Advice by Telephone (CHAT) project. Health Educ Res 2002, 17:627-636.

52. Newman MA, Pettee KK, Storti KL, Richardson CR, Kuller LH, Kriska AM: Monthly variation in physical activity levels in postmenopausal women. Med Sci Sports Exerc 2009, 41:322-327.

53. Rose S, Lawton B, Elley CR, Dowell A, Fenton A: The 'Women's Lifestyle Study', 2-year randomized controlled trial of physical activity counselling in primary health care: rationale and study design. BMC Public Health 2007, 7:166.

54. Lindström J, Eriksson JG, Valle T, Aunola S, Cepaitis Z, Hakumäki M, Hämäläinen $H$, Ilanne-Parikka P, Keinänen-Kiukaanniemi S, Laakso M, Louheranta A, Mannelin M, Martikkala V, Moltchanov V, Rastas M, Salminen V, Sundvall J, Uusitupa M, Tuomilehto J: Prevention of diabetes mellitus in subjects with impaired glucose tolerance in the Finnish Diabetes Prevention Study: results from a randomized clinical trial. J Am Soc Nephrol 2003, 14:S108-113.

55. Lindström J, Absetz $P$, Hemio K, Peltomaki P, Peltonen M: Reducing the risk of type 2 diabetes with nutrition and physical activity - efficacy and implementation of lifestyle interventions in Finland. Public Health Nutr 2010, 13:993-999.

56. Tuomilehto J, Lindström J, Eriksson JG, Valle $\Pi$, Hämäläinen $H$, llanneParikka P, Keinänen-Kiukaanniemi S, Laakso M, Louheranta A, Rastas M, Salminen V, Uusitupa M, Finnish Diabetes Prevention Study Group: Prevention of type 2 diabetes mellitus by changes in lifestyle among subjects with impaired glucose tolerance. N Engl J Med 2001, 344:1343-1350.

57. Crain AL, Martinson BC, Sherwood NE, O'Connor PJ: The long and winding road to physical activity maintenance. Am J Health Behav 2010, 34:764-775.

58. Opdenacker J, Delecluse C, Boen F: The longitudinal effects of a lifestyle physical activity intervention and a structured exercise intervention on 
physical self-perceptions and self-esteem in older adults. J Sport Exerc Psychol 2009, 31:743-760.

59. van Keulen HM, Mesters I, Brug J, Ausems M, Campbell M, Resnicow K, Zwietering PJ, van Breukelen G, van Mechelen W, Severens JL, de Vries H: Vitalum study design: RCT evaluating the efficacy of tailored print communication and telephone motivational interviewing on multiple health behaviors. BMC Public Health 2008, 8:216.

60. Yates T, Davies MJ, Gorely T, Talbot D, Bull F, Sattar N, Khunti K: The effect of increased ambulatory activity on markers of chronic low-grade inflammation: evidence from the PREPARE programme randomized controlled trial. Diabet Med 2010, 27:1256-1263.

61. Eriksson J, Lindström J, Valle T, Aunola S, Hämäläinen H, llanne-Parikka P, Keinänen-Kiukaanniemi S, Laakso M, Lauhkonen M, Lehto P, Lehtonen A, Louheranta A, Mannelin M, Martikkala V, Rastas M, Sundvall J, Turpeinen A, Viljanen T, Uusitupa M, Tuomilehto J: Prevention of Type II diabetes in subjects with impaired glucose tolerance: the Diabetes Prevention Study (DPS) in Finland. Study design and 1-year interim report on the feasibility of the lifestyle intervention programme. Diabetologia 1999, 42:793-801.

62. Monninkhof E, Peeters P, Schuit A: Design of the sex hormones and physical exercise (SHAPE) study. BMC Public Health 2007, 7:232.

63. Racette SB, Weiss EP, Villareal DT, Arif H, Steger-May K, Schechtman KB, Fontana L, Klein S, Holloszy JO, The Washington University School of Medicine CALERIE Group: One year of caloric restriction in humans: feasibility and effects on body composition and abdominal adipose tissue. J Gerontol A Biol Sci Med Sci 2006, 61:943-950.

64. Sherwood NE, Martinson BC, Crain AL, Hayes MG, Pronk NP, O'Connor PJ: A new approach to physical activity maintenance: rationale, design, and baseline data from the Keep Active Minnesota Trial. BMC Geriatr 2008, 8:17.

65. Werkman A, Schuit AJ, Kwak L, Kremers SP, Visscher TL, Kok FJ, Schouten EG: Study protocol of a cluster randomised controlled trial investigating the effectiveness of a tailored energy balance programme for recent retirees. BMC Public Health 2006, 6:293.

66. Yates T, Davies M, Gorely T, Bull F, Khunti K: Rationale, design and baseline data from the Pre-diabetes Risk Education and Physical Activity Recommendation and Encouragement (PREPARE) programme study: a randomized controlled trial. Patient Educ Couns 2008, 73:264-271.

67. Richardson CR, Newton TL, Abraham JJ, Sen A, Jimbo M, Swartz AM: A meta-analysis of pedometer-based walking interventions and weight loss. Ann Fam Med 2008, 6:69-77.

68. Bravata DM, Smith-Spangler C, Sundaram V, Gienger AL, Lin N, Lewis R, Stave CD, Olkin I, Sirard JR: Using pedometers to increase physical activity and improve health: a systematic review. JAMA 2007, 298:2296-2304.

69. Prince SA, Adamo KB, Hamel ME, Hardt J, Connor Gorber S, Tremblay M: A comparison of direct versus self-report measures for assessing physical activity in adults: a systematic review. Int I Behav Nutr Phys Act 2008, 5:56.

70. Greaves CJ, Sheppard KE, Abraham C, Hardeman W, Roden M, Evans PH, Schwarz P, IMAGE Study Group: Systematic review of reviews of intervention components associated with increased effectiveness in dietary and physical activity interventions. BMC Public Health 2011, 11:119.

71. Gebel K, Bauman A, Owen N: Correlates of non-concordance between perceived and objective measures of walkability. Ann Behav Med 2009, 37:228-238.

72. King WC, Belle SH, Brach JS, Simkin-Silverman LR, Soska T, Kriska AM: Objective measures of neighborhood environment and physical activity in older women. Am J Prev Med 2005, 28:461-469, Erratum: Am J Prev Med 2005, 29: 245-246

73. Dwyer T, Ponsonby AL, Ukoumunne OC, Pezic A, Venn A, Dunstan D, Barr E, Blair S, Cochrane J, Zimmet P, Shaw J: Association of change in daily step count over five years with insulin sensitivity and adiposity: population based cohort study. BMJ 2011, 342:c7249.

74. Netz Y, Wu MJ, Becker BJ, Tenenbaum G: Physical activity and psychological well-being in advanced age: a meta-analysis of intervention studies. Psychol Aging 2005, 20:272-284.

75. Angevaren M, Aufdemfampe G, Verhaar HJ, Aleman A, Vanhees L: Physical activity and enhanced fitness to improve cognitive function in older people without known cognitive impairment. Cochrane Database Syst Rev 2008, 16:CD005381.

76. Chan CB, Ryan DA, Tudor-Locke C: Health benefits of a pedometer-based physical activity intervention in sedentary workers. Prev Med 2004, 39:1215-1222.
77. Dwyer T, Hosmer D, Hosmer T, Venn AJ, Blizzard CL, Granger RH, Cochrane JA, Blair SN, Shaw JE, Zimmet PZ, Dunstan D: The inverse relationship between number of steps per day and obesity in a population-based sample - the AusDiab study. Int J Obesity 2007, 31:797-804.

78. Tudor-Locke C, Craig CL, Brown WJ, Clemes SA, De Cocker K, Giles-Corti B, Hatano Y, Inoue S, Matsudo SM, Mutrie N, Oppert JM, Rowe DA, Schmidt MD, Schofield GM, Spence JC, Teixeira PJ, Tully MA, Blair SN: How many steps/day are enough? For adults. Int J Behav Nutr Phys Act 2011, $8: 79$.

79. Wen CP, Wai JPM, Tsai MK, Yang YC, Cheng TYD, Lee M-C, Chan HT, Tsao CK, Tsai SP, Wu X: Minimum amount of physical activity for reduced mortality and extended life expectancy: a prospective cohort study. Lancet 2011, 378:1244-1253.

80. Liberati A, Altman DG, Tetzlaff J, Mulrow C, Gøtzsche PC, loannidis JPA, Clarke M, Devereaux PJ, Kleijnen J, Moher D: The PRISMA statement for reporting systematic reviews and meta-analyses of studies that evaluate healthcare interventions: explanation and elaboration. BMJ 2009, 339: b2700.

\section{Pre-publication history}

The pre-publication history for this paper can be accessed here: http://www.biomedcentral.com/1741-7015/11/75/prepub

\section{doi:10.1186/1741-7015-11-75}

Cite this article as: Hobbs et al: Are behavioral interventions effective in increasing physical activity at 12 to 36 months in adults aged 55 to 70 years? A systematic review and meta-analysis. BMC Medicine 2013 $11: 75$.

\section{Submit your next manuscript to BioMed Central and take full advantage of:}

- Convenient online submission

- Thorough peer review

- No space constraints or color figure charges

- Immediate publication on acceptance

- Inclusion in PubMed, CAS, Scopus and Google Scholar

- Research which is freely available for redistribution 